\title{
Enhanced Electron Pairing in a Lattice of Berry Phase Molecules
}

\author{
Nicola Manini* \\ International School for Advanced Studies (SISSA), via Beirut 2-4, I-34013 Trieste, Italy \\ Erio Tosatti ${ }^{\dagger}$ \\ International School for Advanced Studies (SISSA), via Beirut 2-4, I-34013 Trieste, Italy \\ and International Centre for Theoretical Physics (ICTP), P.O. BOX 586, I-34014 Trieste, Italy \\ Sebastian Doniach ${ }^{\ddagger}$ \\ Dept. Applied Physics, Stanford University, Stanford CA 94305 USA
}

(July 07, 1994)

\begin{abstract}
We show that electron hopping in a lattice of molecules possessing a Berry phase naturally leads to pairing. Our building block is a simple molecular site model inspired by $\mathrm{C}_{60}$, but realized in closer similarity with $\mathrm{Na}_{3}$. In the resulting model electron hopping must be accompanied by orbital operators, whose function is to switch on and off the Berry phase as the electron number changes. The effective hamiltonians (electron-rotor and electron-pseudospin) obtained in this way are then shown to exhibit a strong pairing phenomenon, by means of $1 \mathrm{D}$ linear chain case studies. This emerges naturally from numerical studies of small $N$-site rings, as well as from a BCS-like mean-field theory
\end{abstract}

\footnotetext{
*Email: manini@sissa.it

${ }^{\dagger}$ Email: tosatti@sissa.it

${ }^{\ddagger}$ Email: doniach@drizzle.stanford.edu
} 
formulation. The pairing may be explained as resulting from the exchange of singlet pairs of orbital excitations, and is intimately connected with the extra degeneracy implied by the Berry phase when the electron number is odd. The relevance of this model to fullerides, to other molecular superconductors, as well as to present and future experiments, is discussed.

PACS numbers: 71.27.+a,74.20.Mn,74.70.Wz 


\section{INTRODUCTION}

A significant feature of the physics of unconventional superconductors such as the cuprates is the constraint imposed on the motion of the charge carriers by the background degrees of freedom, i.e. the spins in the case of the cuprate superconductors.

In this paper we focus attention on a new class of constraints imposed on the motion of conduction electrons by the Berry phase [1], or molecular Aharonov-Bohm phase [2], which can arise in molecular crystals with large on-site degeneracies. In general, for this to be the case the symmetries of electron and vibron states must match appropriately and, moreover, the number of electrons needs to be odd.

As an explicit example, we have demonstrated elsewhere [3] the presence of a Berry phase in negatively charged fullerene ions $\mathrm{C}_{60}^{-}, \mathrm{C}_{60}^{3-}, \mathrm{C}_{60}^{5-}$ ensuing from a Dynamic JahnTeller (DJT) effect arising from coupling between the partly occupied $t_{1 u}$ orbital and the $H_{g}$ vibron modes.

In such a situation a physical electron (or hole) may be regarded as a composite particle, made up of the bare electron plus the geometrical phase which accompanies it when sitting, unpaired, (or more generally in a state with an odd number of electrons) on a given molecule. By contrast, either the absence of electrons or presence of a pair of electrons will eliminate the Berry phase on that molecule. We argue below that the kinematical constraints imposed by the Berry phase can be a factor capable of tilting the balance in favor of pairing, even in the presence of repulsive interactions [四]. The way this works is for a pair of electrons on one molecule to gain energy by each tunnelling off onto neighbors with accompanying vibron excitations before coming together again. A single electron on the other hand will tunnel accompanied by its vibron excitations, so the electrons will gain less energy by tunnelling as individuals than will be gained by tunnelling as pairs.

In this paper, we propose a class of simple coupled electron-rotor models which we believe capture the essential physics introduced by the Berry phase constraint. Omitting at this stage the complications of real $\mathrm{C}_{60}^{n-}$ anions, our model lattice hamiltonian is instead directly 
inspired by the simpler and well known [5] strong-coupling Berry phase molecule $\mathrm{Na}_{3}$.

In general there are an infinite number of rotor states on each molecular site. To simplify further, we shall truncate to a 3-state model for the rotors. The resulting many-site Hamiltonian will be shown to take the form

$$
H=-\frac{t}{2} \sum_{<i, j>, \sigma} c_{i, \sigma}^{\dagger} c_{j, \sigma}\left(S_{i}^{+} S_{j}^{-}+\text {h.c. }\right)
$$

where $S^{+}, S^{-}$are raising and lowering operators for a spin-1 vibron manifold for each molecule, and $<i, j>$ denote first neighbors.

To test the pairing properties of (1), we include a Hubbard repulsion term

$$
H_{U}=U \sum_{i} n_{i \uparrow} n_{i \downarrow}
$$

We then study the half-filled state of the model numerically for small systems (4 to 8 site rings), and also by a mean field BCS-type calculation for the 1-D chain.

The final conclusion is that the Berry phase coupling is found to be greatly beneficial to electron pairing, at least within the simple 1D lattice studied so far. Pairing, in particular, appears to prevail and to survive even in presence of a repulsive Hubbard $U$, up to values $U \approx t$.

In Section II we will introduce the main concepts and build our working hamiltonian, representing an idealized lattice of Berry phase molecules.

Section III is devoted to a numerical study of the 4, 6 and 8-site 1D chain clusters, where correlations can be studied exactly, and the presence of pairing is demonstrated.

Section $\mathbb{\square}$ discusses a mean field BCS-type formulation for the infinite 1-D chain.

We close with a discussion section, where a number of interesting open issues are also briefly presented.

\section{THE MODEL}

Our model system is a regular lattice of molecules. Each molecule possesses initially a degenerate orbital, an odd number of electrons, and a dynamical Jahn-Teller (DJT) coupling 
(with Berry phase) to a local vibration, also degenerate, of pseudorotational type. For simplicity, we stick to the case of one electron in a doubly degenerate orbital and one rotor, which provides the simplest case of Berry phase coupling. The electron can hop from a molecule to the next one in the usual fashion, conserving ordinary spin. We will generally also include an on-site ("intra-molecular") electron-electron repulsion $U$, so that,

for a nondegenerate level and no rotor coupling, we would have just an ordinary Hubbard model. Berry phase coupling to the rotors is the new ingredient giving rise to peculiar selection rules for electron hopping between two molecules in different rotor states. In the following, we describe successively the on-site hamiltonian, and the full lattice hamiltonian inclusive of electron hopping.

\section{A. On-site hamiltonian: modeling a Berry phase molecule}

We consider at each site a molecule with a partly occupied doubly degenerate electronic state. Suppose this orbital interacts via linear DJT coupling with a doubly degenerate vibration. A practical example (not relevant for superconductivity) of such a situation is the $\mathrm{Na}_{3}$ molecule. A single unpaired electron occupies the doubly degenerate electronic molecular orbital $E \equiv\left(E_{x}, E_{y}\right)$, and the doubly degenerate vibron is a pseudorotation of the $\mathrm{Na}_{3}$ triangular structure. Direct spectroscopical evidence has been found [5], showing that the formalism we present here (supplemented by quadratic couplings which are omitted here for simplicity) correctly describes the dynamics of this system.

The single-molecule, one-electron, linear coupling case, is a classic Jahn-Teller textbook problem [6]. When only one electron occupies the degenerate state, the lowest order electronvibron hamiltonian can be written:

$$
H=\frac{\hbar \omega}{2}\left[\frac{\partial^{2}}{\partial q_{1}^{2}}+\frac{\partial^{2}}{\partial q_{2}^{2}}+q_{1}^{2}+q_{2}^{2}\right]\left(\begin{array}{ll}
1 & 0 \\
0 & 1
\end{array}\right)+g \frac{\hbar \omega}{2}\left(\begin{array}{cc}
-q_{1} & q_{2} \\
q_{2} & q_{1}
\end{array}\right)
$$

where the $2 \times 2$ matrices span the twofold $E$ electronic level, and $q_{i}$ are the vibrational normal coordinates in the vibron space; the second term is the coupling between vibron and electron, of dimensionless strength $g$. 
The problem is rotationally invariant in the $\left(q_{1}, q_{2}\right)$ space. It is therefore conveniently rewritten by introducing polar coordinates $q, \phi$ in the vibron space and a pseudospin $\frac{1}{2}$ representation in the electron space:

$$
\left|\mp \frac{1}{2}\right\rangle= \pm \frac{i}{\sqrt{2}}\left(\left|E_{x}\right\rangle \mp i\left|E_{y}\right\rangle\right)
$$

In terms of these quantities the hamiltonian is expressed as:

$$
H=\frac{\hbar \omega}{2}\left[-\frac{\partial^{2}}{\partial q^{2}}-\frac{1}{q} \frac{\partial}{\partial q}-\frac{1}{q^{2}} \frac{\partial^{2}}{\partial \phi^{2}}+q^{2}\right]+\frac{g \hbar \omega}{2}\left(\begin{array}{cc}
0 & q e^{+i \phi} \\
q e^{-i \phi} & 0
\end{array}\right)
$$

A new total angular momentum $j$, defined as

$$
j=\hbar^{-1}\left(q_{1} p_{2}-q_{2} p_{1}\right)-\frac{1}{2} \sigma_{z}=-i \frac{\partial}{\partial \phi}-\left(\begin{array}{cc}
\frac{1}{2} & 0 \\
0 & -\frac{1}{2}
\end{array}\right),
$$

is found to commute with $H$ [6]. Note that $\sigma_{z}$ is only a pseudospin $\frac{1}{2}$ spanning in reality the twofold orbital state, and should not be confused with the true spin, which is ignored at this stage. Because of this pseudospin $\frac{1}{2}$ term, the eigenvalues of $j$ are half-odd integer, an amusing anomaly first pointed out by Herzberg and Longuet-Higgins []] for the case of the triangular molecule. This fractionalization can be seen as a manifestation of a Berry phase [1] of $\pi$, which the vibrons pick up from the electron degeneracy [5].

Diagonalization of (5) must in general be done numerically [6,8]. In the limit of strong coupling $(g>>1)$, however, the massive radial $q$-motion can be approximately separated from the $\phi$-pseudorotation quantized by $j$, and both can be solved analytically in the form of an oscillator and a free rotor respectively.

The resulting spectrum is classified according to $j$ and $\nu$, the quantum number coming from the quantization of the radial massive motion motion:

$$
E(v, j)=\hbar \omega\left(\nu+\frac{1}{2}\right)+\frac{2 \hbar \omega}{g^{2}}\left(j^{2}+\frac{1}{4} \pm \sqrt{j^{2}+\frac{g^{8}}{64}}\right), \nu=0,1,2, \ldots, j= \pm \frac{1}{2}, \pm \frac{3}{2}, \ldots
$$

Since we shall be concerned only with the low lying rotor states, we can forget the massive boson ladder. Furthermore, we will express all energies in units of the pseudorotational quantum $\Omega:=\frac{2 \hbar \omega}{g^{2}}$. 
The corresponding wavefunctions in the strong coupling limit are:

$$
\psi_{\nu, j}(q, \phi)=\psi_{\nu}(q)\left(\begin{array}{c}
\cos \theta_{j} e^{i\left(j+\frac{1}{2}\right) \phi} \\
\sin \theta_{j} e^{i\left(j-\frac{1}{2}\right) \phi}
\end{array}\right) j \text { half-odd integer }
$$

where $\psi_{\nu}(q)$ is the appropriate harmonic oscillator wavefunction, and $\theta_{j}$ is a pseudospin mixing angle

$$
\tan \theta_{j}=8 \frac{-j \pm \sqrt{j^{2}+\frac{g^{8}}{64}}}{g^{4}}
$$

In the $g \rightarrow \infty$ (strong coupling) limit $\theta_{j}$ tends to $\pm \frac{\pi}{4}$. The energy can be expanded as

$$
E(j)= \pm \frac{g^{4}}{8}+j^{2}+\frac{1}{4} \pm 4 \frac{j^{2}}{g^{4}}+\cdots
$$

At low energies, we consider only the rotor states

$$
\psi_{j}(\phi)=2^{-\frac{1}{2}}\left(\begin{array}{c}
e^{i\left(j+\frac{1}{2}\right) \phi} \\
-e^{i\left(j-\frac{1}{2}\right) \phi}
\end{array}\right), j= \pm \frac{1}{2}, \pm \frac{3}{2}, \pm \frac{5}{2}, \ldots
$$

with energy:

$$
E(j)=j^{2}, \quad j= \pm \frac{1}{2}, \pm \frac{3}{2}, \pm \frac{5}{2}, \ldots
$$

where we have omitted as usual the $-\frac{g^{4}}{8}$ offset contribution (polaron energy shift), but also the extra zero point energy $1 / 4$ required by the Berry phase [3]. This is our simplified model for the one-electron Berry molecule.

To study electron hopping among different molecules, we also need an equivalent description for molecular occupancy different from one. When two electrons occupy the molecular orbital in a singlet state, the DJT distortion is still present. However, the orbital phases for the two electrons cancel each other and a Berry phase is no longer present.

For uniformity with the one-electron case, we will still label for $n=2$ the pseudorotational levels with $j$. Cancellation of the geometrical phase now requires $j$ to be integer. The assumed spectrum is simply that of a standard free pseudorotor:

$$
E(j)=j^{2}, j=0, \pm 1, \pm 2, \ldots
$$


with wavefunctions

$$
\psi_{j}(\phi)=e^{i j \phi}, j=0, \pm 1, \pm 2, \ldots
$$

In the energy eigenvalue (13) we are again omitting the polaron energy gain $-\frac{g^{4}}{2}$, (units of $\Omega)$.

In the $n=0$ case where no electron is present, no Jahn-Teller effect and thus no pseudorotor either. However we really would like to mimic an electron-hole symmetric situation, like for example going $\mathrm{C}_{60}^{2-}$ or $\mathrm{C}_{60}^{4-}$ with respect to $\mathrm{C}_{60}^{3-}$. For this reason we assume even for zero electrons a pseudorotation (13, 14) identical to that of the two electron case.

We finally discard occupancies higher than two; for instance, we may suppose that, if the average occupancy is one, states with $n>2$ are strongly suppressed by Coulomb repulsions.

To summarize, if $n$ is the number of electrons present in the degenerate orbital, $2 j$ assumes even or odd values according to whether $n$ is even or odd. The $j$-dependence of the energy eigenvalues is quadratic, like in a free (pseudo-)rotor. The full quantum state of such a molecule, in the limit considered, is described by a set of three quantum numbers which for convenience we define as integers:

$$
\begin{array}{r}
n,(\text { occupancy }) \\
m=2 j,(\text { rotor state) } \\
\sigma=2 m_{s},(\text { spin state) }
\end{array}
$$

where $m_{s}= \pm \frac{1}{2}$ is the $\mathrm{z}$ component of true electron spin. Their allowed values are constrained in the form:

$$
\begin{aligned}
& n=0,1,2 \\
& m=2 l+\sin (\pi n), l=0, \pm 1, \pm 2, \pm 3 \cdots \\
& \sigma= \pm \sin (\pi n)
\end{aligned}
$$

With reference to the physics of $\mathrm{C}_{60}^{n-}$, discussed in Ref. [3], the similarity with the present model should be clear. In that case, in particular, the ground state has even $L$ for even $n$, and odd $L$ for odd $n$. 


\section{B. Inter-site hamiltonian $\rightarrow$ hopping between Berry molecules.}

To allow electrons to move among sites, we need to specify how the hopping process is affected by the $j$ quantum numbers on each site. Therefore we begin considering one electron hopping between two neighbouring molecules. With sufficiently high point symmetry, such

as one has for the linear chain, we will have hopping between $\left|E_{x, 1}\right\rangle$ and $\left|E_{x, 2}\right\rangle$ and between $\left|E_{y, 1}\right\rangle$ and $\left|E_{y, 2}\right\rangle$ only, with amplitude $t_{x}$ and $t_{y}$ respectively [9]. If for instance the twofold degenerate state is associated to a p-orbital, then $t_{x}=t_{p p \sigma}, t_{y}=t_{p p \pi}$, in Slater-Koster's notation [10]. The hopping hamiltonian then is

$$
H_{k i n}=\sum_{\sigma}\left[t_{x}\left(c_{x, \sigma, 1}^{\dagger} c_{x, \sigma, 2}+\text { h.c. }\right)+t_{y}\left(c_{y, \sigma, 1}^{\dagger} c_{y, \sigma, 2}+\text { h.c. }\right)\right]
$$

To characterize its behaviour, suppose we have on site 2 a spin up electron, with $m_{2}$ (odd) molecular pseudospin, and we want the hopping amplitude of this electron to site 1 with final spin up, and pseudospin $m_{1}$ (also odd). We need to invert relation (雨) to express the fermionic operators in the pseudospin basis:

$$
\begin{aligned}
& c_{x, s}^{\dagger}=\frac{c_{+, s}^{\dagger}-c_{-, s}^{\dagger}}{-i \sqrt{2}} \\
& c_{y, s}^{\dagger}=\frac{c_{+, s}^{\dagger}+c_{-, s}^{\dagger}}{\sqrt{2}}
\end{aligned}
$$

The matrix element we compute depends also on the (even) pseudospin of the empty sites $l_{2}$ (final state) and $l_{1}$ (initial state) in the following way:

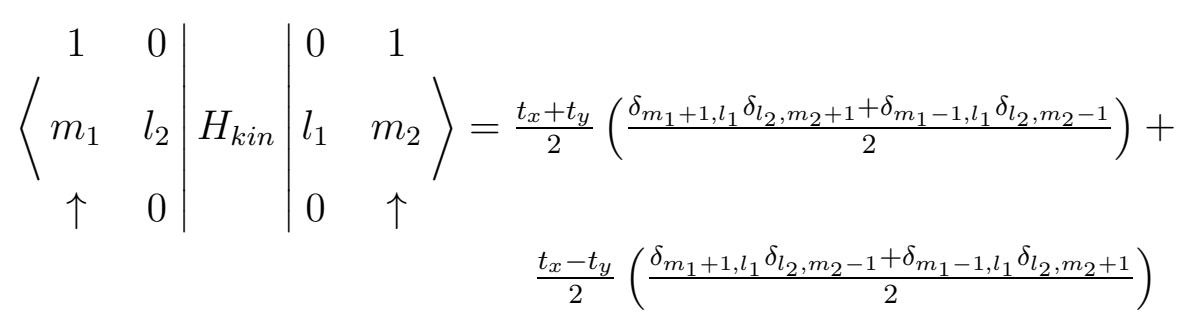

The deltas here originate through trivial orthogonality of angular momentum wavefunctions (11, 14), while the overall $\frac{1}{2}$ factors originate from the $\sqrt{2}$ factors in (18) and in (11).

If we limit ourselves to the special case of intermolecular interaction with $t_{y}=t_{x}\left(t_{y}=\right.$ $\left.-t_{x}\right)$, then we have an additional conservation $l_{1}+m_{2}=m_{1}+l_{2}\left(l_{1}-m_{2}=m_{1}-l_{2}\right)$ of the 
total pseudospin, as shown by Eq. (19). In this cases, the spectrum separates into different independent manifolds. For $t_{y}=t_{x}$, they correspond to different values of total pseudospin. Even if somewhat unrealistic (for a real p state, $t_{x}$ is larger and positive, $t_{y}$ smaller and negative) this is a very convenient choice and we shall adopt it in the following. We have made a check, to be described in Sec. III, releasing this restriction, which have satisfied us that the physics is not fundamentally different in the more general case $t_{y} \neq t_{x}$. Accordingly, we define a single effective hopping

$$
t=\frac{t_{x}+t_{y}}{2}
$$

which in the absence of direct electron-electron interactions is the only independent parameter of our model, the rotor energy quantum $\Omega$ being unity.

Similar considerations and selection rules to those discussed above for the process $|0,1>\rightarrow| 1,0>$ apply to the case involving doubly occupied sites, namely $|0,2>\rightarrow| 1,1>$, or $|1,2>\rightarrow| 2,1>$.

Now we have all the ingredients to place these "Berry molecules" on a lattice to see the effect of local rotor coupling on electron hopping.

\section{Lattice of Berry molecules: the working hamiltonian}

As a first attempt to study this model on a lattice we consider a linear chain of $N$ sites, with $N_{e l}$ electrons moving on them. The basis we consider is labelled by the set of all the $n_{i}, m_{i}, \sigma_{i}$, for $i=1, \cdots, N$, so that an arbitrary state is expandable on states:

$$
\left|\begin{array}{cccc}
n_{1} & n_{2} & \cdots & n_{N} \\
m_{1} & m_{2} & \cdots & m_{N} \\
\sigma_{1} & \sigma_{2} & \cdots & \sigma_{N}
\end{array}\right\rangle .
$$

These basis states are obtained by ordered applications of $N_{e l}$ local fermionic creation op-

erators $c_{j, \sigma}^{\dagger}$ on a vacuum state where no electrons are present, and setting the $m_{i}$ rotational quantum numbers to values allowed by the Berry constraint (16). 
Hopping of an electron between site $i$ and site $j$ is, in this space, a composite operation, since it implies $n_{i} \rightarrow n_{i}-1, n_{j} \rightarrow n_{j}+1$, but also $m_{i} \rightarrow m_{i} \pm 1, m_{j} \rightarrow m_{j} \mp 1$ (16, 19). Hence, we write a general hopping hamiltonian in second quantized language as:

$$
H_{k i n}=-\frac{t}{2} \sum_{<i, j>, \sigma} c_{i, \sigma}^{\dagger} c_{j, \sigma}\left(L_{i}^{+} L_{j}^{-}+L_{i}^{-} L_{j}^{+}\right)
$$

where the action of the operator $L_{j}^{ \pm}$is to raise (lower) the pseudospin $m_{j}$ (really an orbital angular momentum) by one unit:

$$
L_{j}^{ \pm}\left|\begin{array}{cccc}
n_{1} & \cdots & n_{j} & \cdots \\
m_{1} & \cdots & m_{j} & \cdots \\
\sigma_{1} & \cdots & \sigma_{j} & \cdots
\end{array}\right\rangle=\left|\begin{array}{cccc}
n_{1} & \cdots & n_{j} & \cdots \\
m_{1} & \cdots & m_{j} \pm 1 & \cdots \\
\sigma_{1} & \cdots & \sigma_{j} & \cdots
\end{array}\right\rangle .
$$

To the hopping hamiltonian $H_{k i n}$, we add an on-site rotor hamiltonian $H_{\text {rot }}$, as well as an additional on-site electron-electron Hubbard interaction term $H_{U}$

$$
\begin{aligned}
H_{\text {rot }} & =\sum_{j=1}^{N} \frac{1}{4}\left(L_{j}^{z}\right)^{2} \\
H_{U} & =U \sum_{j=1}^{N} n_{j, \uparrow} n_{j, \downarrow},
\end{aligned}
$$

the rotor energy contribution is at site $j$ due in the rotor state $m_{j}$ being

$$
H_{\text {rot } j}=\frac{1}{4} m_{j}^{2}
$$

as in Eq. (12,13), and having introduced a third operator

$$
L_{j}^{z}\left|\begin{array}{cccc}
n_{1} & \cdots & n_{j} & \cdots \\
m_{1} & \cdots & m_{j} & \cdots \\
\sigma_{1} & \cdots & \sigma_{j} & \cdots
\end{array}\right\rangle=m_{j}\left|\begin{array}{cccc}
n_{1} & \cdots & n_{j} & \cdots \\
m_{1} & \cdots & m_{j} & \cdots \\
\sigma_{1} & \cdots & \sigma_{j} & \cdots
\end{array}\right\rangle .
$$

The commutation relations for these operators are $\left[L^{+}, L^{-}\right]=0,\left[L^{z}, L^{ \pm}\right]= \pm L^{ \pm}$.

We stress here that the kinetic term $H_{k i n}$ alone in Eq. (22) is the relevant part of the new hamiltonian we want to study.

The new electron operators $c_{i, \sigma}^{\dagger} L_{i}^{ \pm}$are diffrent from the original ones, $c_{x, \sigma, i}^{\dagger}, c_{y, \sigma, i}^{\dagger}$ of Eq. (17). In particular, we have now a single band, instead of the original double band problem. 
However, with the exclusion of all site occupancies higher than 2, all matrix elements are the same in the two descriptions.

By construction, the hamiltonian (22) conserves the constraints (16) among the quantum numbers, and can therefore be diagonalized in the Hilbert space of states defined in (21). The matrix elements of the kinetic term $H_{k i n}$ (off diagonal) and of $H_{r o t}+H_{U}$ (diagonal) on the basis (21) are trivial, once periodic boundary conditions (PBC) are applied to indexes. In some cases we shall however need antiperiodic boundary conditions (ABC), replacing $t$ with $-t$ in the kinetic term involving sites 1 and $N$.

The Hilbert space of the problem is infinite-dimensional even for finite $N$, due to the $m_{i}$ rotor quantum numbers, which are boundless. In the numerical computations, we shall truncate the basis (21) by choosing a cutoff energy $E_{c u t}$, including only states having some local energy smaller than this $E_{c u t}$. Unfortunately, the choice $H_{r o t} \leq E_{\text {cut }}$ is unfair with respect to singly occupied states, having larger energy (at least by $1 / 4$ ) than the unoccupied and doubly occupied lowest- $j$ ones. To achieve better convergence even at relatively small $E_{c u t}$, we subtract this contribution and retain those states satisfying:

$$
H_{\text {rot }}+\sum_{j}\left(\frac{n_{j, \uparrow} n_{j, \downarrow}}{2}-\frac{n_{j, \uparrow}+n_{j, \downarrow}}{4}\right) \leq E_{c u t},
$$

The special case $E_{c u t}=0$ is of very strong interest. Physically, this corresponds to the limiting case $t<<\Omega$, where the intramolecular rotor energy is much larger than the hopping energy. In this limit, the only allowed values for $m_{j}$ are 0 (even occupancy) and \pm 1 (odd occupancy). The resulting model has six states per site, two corresponding to even ( 0 and 2$)$ occupancies, and four to the $2 \times 2$ combinations of spin $\sigma_{j}$ and pseudospin $m_{j}$ values allowed for one electron. It is possible and convenient to rewrite this simplified version of the model in terms of fictitious spin- 1 states, the $m_{j}$ quantum number becoming the $z$ projection of a pseudospin $S=1$. For this simplified version we can rewrite the hamiltonian (22) replacing the free rotor operators $L^{+}, L^{-}, L^{z}$ with the generators of the spin 1 algebra [11] $S^{+}, S^{-}, S^{z}$ respectively 


$$
H_{k i n}^{\prime}=-\frac{t}{2} \sum_{<i, j>, \sigma} c_{i, \sigma}^{\dagger} c_{j, \sigma}\left(S_{i}^{+} S_{j}^{-}+\text {h.c. }\right)
$$

The full ladder of rotational states (11,14) has now disappeared, being replaced just by the double degeneracy of the $n=1$ sites $\left(S_{z}= \pm 1\right)$, with $H_{\text {rot }} \equiv 0$ for all $n$.

The extra terms (24) would still need to be added to $H_{k i n}^{\prime}$. However, $H_{\text {rot }}$ has the simple effect of giving a energy shift of $\Omega / 4$ per each singly occupied site. As suggested above, this has the same effect of an operator such as:

$$
\Omega \sum_{j}\left(\frac{n_{j, \uparrow} n_{j, \downarrow}}{2}-\frac{n_{j, \uparrow}+n_{j, \downarrow}}{4}\right),
$$

i.e. it is the same as a positive Hubbard $U$ term with $U=\frac{\Omega}{2}$, apart from a chemical potential. For $t<<\Omega$, this term amounts to a divergent shift of the Hubbard $U$. Such a diverging term has no physical origin (the JT energy gains we have neglected in (12) and (13) are also infinite and have opposite sign). Therefore we will omit it, and simply work with Hamiltonian (28), with the only caveat that we need to remember the shift in Hubbard $U$ when comparing the results of this low-cutoff model (28) with the fully converged one.

Both hamiltonians (22) and (28) show a significant degree of symmetry which we can take advantage of. Each of them conserves the number of electrons $N_{e l}$, the total pseudoangular momentum $2 J=M=\sum_{i} m_{i}$ (i. e. the total $S_{z}$ in the $S=1$ pseudospin version), and total electron spin. For a linear chain, the lattice translational symmetry is also obvious. Pseudospin conservation is a result of our approximation $t_{x}=t_{y}$. The others are exact. We choose to study the problem (22) in the manifold at half filling $\left(N_{e l}=N\right)$ and at $M=0$ (even $N$ ), because of the higher symmetry present in this case, which includes electron-hole symmetry. Although we have not yet carried out a complete study of the model away from half filling, we believe that the basic physics will be (at least for $U=0$ ) the same, due to a suggestive analogy with the negative $U$ Hubbard model which will finally emerge.

At this point, we are set with two alternative working models. The electron-rotor (ER) model

$$
H_{E R}=H_{k i n}+H_{\text {rot }}+H_{U}
$$


where $H_{\text {kin }}, H_{\text {rot }}$ and $H_{U}$ are given by (22), (24), is more realistic, and is characterized by two parameters, the hopping energy $t$ and the rotor energy $\Omega$. This latter quantity, in turn, contains the ionic mass, and will therefore make the model sensitive to isotopic changes. The second, electron-pseudospin one (EP) model

$$
H_{E P}=H_{k i n}^{\prime}+H_{U},
$$

where $H_{k i n}^{\prime}$ is given by (28), represents the extreme molecular limit and is more idealized, the hopping energy $t$ being the only parameter. Clearly, there will be no isotope effect in this model.

Although the important terms are $H_{k i n}$ and $H_{k i n}^{\prime}$, both models are endowed with the Hubbard term $H_{U}$, which can describe additional repulsive interactions, and is also convenient as a gauge of the effective attractions which will arise. Having taken $\Omega$ as the energy unit, the physical results in the ER model will depend on the two dimensionless ratios $t / \Omega$, $U / \Omega$. Those in the EP model will depend only upon $U / t$, making direct comparison with the simple Hubbard model particularly straightforward.

As it will be shown, there is numerical evidence that the two models, ER and EP, lead to qualitatively similar effects, at least when $t$ is not too large. Hence, it will be possible for many purposes to focus on the simpler EP model.

In the next two sections, we propose to study these models on a 1D linear chain, as follows. First, we will study numerically some very small clusters, by direct diagonalization. This will permit a first crude comparison between ER and EP, and also between them and the simple Hubbard model. Next, we will introduce a mean-field theory for model EP on the infinite 1D linear chain. Here, the $S=1$ pseudospin variables can be approximately integrated out, giving rise to negative effective electron-electron forward and backward couplings, again suggesting singlet pairing. 


\section{NUMERICAL STUDIES FOR SMALL LINEAR CHAIN CLUSTERS (RINGS)}

We consider here small $N$-site linear chain clusters (rings), in particular $N=4,6,8$, accessible to numerical diagonalization using conventional Lanczos method. While these sizes are admittedly small, we find that the qualitative results for small $N$ are clear enough at this initial stage [12].

We define useful equal-time correlation functions for singlet superconductivity (SC) and spin density wave (SDW) in the standard form of $q$-space "structure factors":

$$
\begin{aligned}
S_{S C}(q) & =\frac{1}{N} \sum_{j, l}^{N} e^{i q(j-l)}\left\langle c_{j, \downarrow}^{\dagger} c_{j, \uparrow}^{\dagger} c_{l, \uparrow} c_{l, \downarrow} \mathbf{I}_{m}\right\rangle \\
S_{S D W}(q) & =\frac{1}{N} \sum_{j, l}^{N} e^{i q(j-l)}\left\langle c_{j, \uparrow}^{\dagger} c_{j, \downarrow} c_{l, \downarrow}^{\dagger} c_{l, \uparrow} \mathbf{I}_{m}\right\rangle
\end{aligned}
$$

We ignore alternative channels, such as charge density waves (CDW) or triplet superconductivity [13], which can also be probed, but whose behaviour is not relevant at this stage. In particular, a CDW will be definitely disfavoured in the more general case away from half filling. The property of correlations (32) and (33) is that they transform into one another

under the transformation $c_{j, \uparrow} \rightarrow \tilde{c}_{j, \uparrow}, c_{j, \downarrow}^{\dagger} \rightarrow(-1)^{j} \tilde{c}_{j, \downarrow}$, which, remarkably, amounts simply to the transformation $U \rightarrow-U$ [14] in the Hubbard model. Hence in that model $S_{S C}(q)$ and $S_{S D W}(q)$ are perfectly symmetric around $U=0$, where their values must cross, the SC instability prevailing for $U<0$, the SDW for $U>0$. This of course is confirmed for the $N$-site rings, as shown in Fig. 11. In the upper panel we choose PBC, so that the free system $(U=0)$ is in a closed shell configuration of 6 electrons. In the lower panel instead ABC are applied. Here, the resulting shift of the single-particle $k$-states yields an open shell for free fermions at half filling. Open shell and closed shell calculations must finally converge to the same answer for $N \rightarrow \infty$, and their systematic comparison at finite $N$ provides a rough but useful measure of finite-size corrections.

Our strategy is therefore to calculate $S_{S C}$ and $S_{S D W}$ for our hamiltonian $H_{E R}$ and $H_{E P}$, as a function of $U / t$, and to find the value of $U=U^{*}$ where they cross, so that superconducting pairing prevails at all $U<U^{*}$. The finding that $U^{*}$ is finally positive will in turn imply 
that the bare $U=0$ model is approximately equivalent to a negative Hubbard $U$ model, with $U=U_{\text {eff }}$, where a crude linear estimate is

$$
U_{e f f} \approx-U^{*}
$$

so long as $U^{*}$ is small.

Figure 2 shows results for the EP model, obtained for $N=4,6,8$ sites, at half filling $\left(N_{e l}=N\right)$ as a function of $U$. The two panels a) and b) correspond to the different choices of closed shells and open shells respectively. For instance, $N=4$ and 8 correspond to closed shells generated with antiperiodic boundary conditions (ABC), while $N=6$ does that with periodic boundary conditions (PBC). Conversely $N=4$ and 8 yield open shells with $\mathrm{PBC}$, $N=6$ with $\mathrm{ABC}$. The condensation wavevector $q$ is correspondingly zero with $\mathrm{PBC}$ and $q=\frac{\pi}{N}$ for $\mathrm{ABC}$.

These results show, strikingly, that in the EP model, a finite positive $U^{*} / t$ is needed to suppress superconductivity in favor of SDW's. Roughly, the EP model (28) behaves therefore like a negative $U$ Hubbard model, with $U_{\text {eff }} \propto-t$ For small $N$, the value of $U_{\text {eff }}$ varies with $N$, and also depends on whether the shell is closed / open. Although we have not tried a systematic finite-size scaling extrapolation for $U_{\text {eff }}$ to the $N=\infty$ limit, the result up to $N=8$ suggests that

$$
-0.8 t<U_{\text {eff }}<-0.2 t
$$

In particular at $N=6$ both $\mathrm{ABC}$ and $\mathrm{PBC}$ yield $U_{\text {eff }}=-0.37|t|$, which may therefore be a likely value.

We have also studied the full ER model (30). For this model, the Hilbert space is that of states (21), with an upper cutoff in the rotor states $E_{\text {cut }}$. Due to the larger Hilbert space, we have restricted calculations to $N=4,6$. We proceed by calculating $S_{S C}$ and $S_{S D W}$ for fixed $t / \Omega$ as a function of $U / \Omega$, and we look for the value $U^{*} / \Omega$ where they cross. This again defines, via (35), a value for $U_{\text {eff }}$. Typical results are shown in Fig. 3 .

Now $t$ is an independent parameter. The effective interaction $U_{\text {eff }}$ can be recalculated by varying $t$, and the results are given in Fig. 4. The main feature is that the negative 
$U_{\text {eff }}$ at small $t$, already found in model EP, is confirmed. Hence, model ER also leads to superconductive pairing for $t / \Omega$ not too large. This fully confirms our expectations that kinematical restrictions imposed by the switching of orbital states are important in that regime. For larger values of $t$, these restrictions gradually become irrelevant, until, for $t \rightarrow \infty$ we recover the value $U_{\text {eff }} \rightarrow 0$. In other words, when the hopping energy is too large, the DJT effect does not work any more, and Fig. 1 describes how its "phase" part is quenched (the JT distortion magnitude is held constant in our model).

One may suspect that the pairing we are demonstrating is just a consequence of the exact symmetry between the $x$ and $y$ degenerate molecular orbitals that we enforce by the assumption of having equal intermolecular hopping matrix elements $t_{x}, t_{y}$, defined in (17), as discussed in Section [1]. A simple test releasing this assumption shows that this is actually not the case. If $t_{x} \neq t_{y}$, the hitherto missing term corresponding to unequal $t_{x}, t_{y}$ term is the following kinetic additive contribution:

$$
H_{k i n}^{\prime}=-\frac{t^{\prime}}{2} \sum_{<i, j>, \sigma} c_{i, \sigma}^{\dagger} c_{j, \sigma}\left(L_{i}^{+} L_{j}^{+}+L_{i}^{-} L_{j}^{-}\right),
$$

where $t^{\prime}$ is the independent hopping amplitude $t^{\prime}=\left(t_{x}-t_{y}\right) / 2$. This term violates the conservation of total $m$ (equivalent to complete $x-y$ symmetry). By adding to the symmetric hamiltonian (30) a term like (36) we can break continuously this symmetry, monitoring the effects of this on pairing, in particular on the $U_{\text {eff }}$ that was defined above.

In Fig. [ 5 we plot $U_{\text {eff }}$ for the EP model (for simplicity) in the closed shell configurations for 4 and 6 sites, as a function of $t / t^{\prime}$, at fixed $t+t^{\prime}=t_{x}$. This figure shows that although the negative effective attractive $U_{\text {eff }}$ is maximum for $t_{x}=t_{y}$ and $t_{x}=-t_{y}$, it is not cancelled in the general case $t_{x} \neq t_{y}$, except for the very special case $t_{y}=0$ (or $t_{x}=0$ ). The cancellation of pairing interaction in this limit is due to the complete breaking of the rotational symmetry, creating two separate bands from the $x$ and $y$ orbitals, the $x$-originated being a regular tightbinding band, the $y$ one being made of localized degenerate states. Anyway, this is indeed a very special case, in which no $t_{y}$ term is present. In a more realistic situation having, say, $t_{y} \approx-t_{x} / 2$, the pairing effect fully survives. 


\section{THE INFINITE CHAIN - MEAN FIELD BCS APPROACH.}

In order to get a qualitative idea of the effects of the Berry phase constraints on the infinite system, we first integrate out the vibron degrees of freedom in the simple spin-

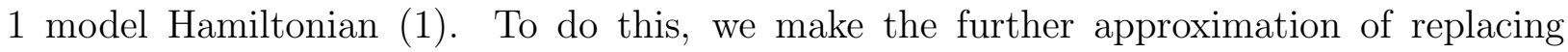
the spin operators by pseudo-fermion operators representing the vibron excitations. We can then integrate out the vibron degrees of freedom and apply the BCS equations to the resulting interacting fermion system.

Introducing an auxiliary spin- $\frac{1}{2}$ fermion $B_{i \alpha}^{\dagger}$, we classify the $m=+1$ vibron state as an " $\alpha$-up" state, and the $m=-1$ state as an " $\alpha$-down" state. The $m=0$ state is treated as a vacuum state for the $B$-fermions, which we will call "berryons":

$$
\left|m=1>\equiv B_{\uparrow}^{\dagger}\right| 0>,\left|m=-1>\equiv B_{\downarrow}^{\dagger}\right| 0>.
$$

Using this representation, we express the spin- 1 operators by

$$
S^{+} \equiv B_{\uparrow}^{\dagger}+B_{\downarrow}, S^{-} \equiv B_{\uparrow}+B_{\downarrow}^{\dagger}, S^{z} \equiv B_{\uparrow}^{\dagger} B_{\uparrow}-B_{\downarrow}^{\dagger} B_{\downarrow}
$$

This representation is overcomplete (in particular it does not exclude unphysical states with $n_{i}, m_{i}$ of different parity), and will therefore not allow a strictly variational treatment. Still, it is of use in exploring whether the model does or does not display tendencies toward pairing at the simplest mean field level.

We rewrite the hamiltonian (11) in this fermion representation as:

$$
H_{k i n}=-\frac{t}{2} \sum_{<i, j>, \sigma} c_{i, \sigma}^{\dagger} c_{j, \sigma}\left(B_{i \uparrow}^{\dagger} B_{j \uparrow}+B_{i \downarrow}^{\dagger} B_{j \downarrow}+B_{i \uparrow}^{\dagger} B_{j \downarrow}^{\dagger}+B_{i \downarrow} B_{j \uparrow}+h . c .\right)
$$

or, in Fourier representation,

$$
\begin{aligned}
H_{k i n}= & -\frac{t}{2 N} \sum_{k_{1}, k_{2}, q, \sigma} \cos \left(k_{1}+q\right) c_{k_{1}, \sigma}^{\dagger} c_{k_{2}, \sigma} \\
& \left(B_{q \uparrow}^{\dagger} B_{k_{1}-k_{2}+q \uparrow}+B_{q \downarrow}^{\dagger} B_{k_{1}-k_{2}+q \downarrow}+B_{q \uparrow}^{\dagger} B_{-k_{1}+k_{2}-q \downarrow}^{\dagger}+B_{-q \downarrow} B_{k_{1}-k_{2}+q \uparrow}+\text { h.c. }\right) .
\end{aligned}
$$

We take as a zeroth order mean field hamiltonian just free fermions (note that this term was missing in the original problem) 


$$
H_{M F}=\sum_{k, \sigma} \epsilon_{k} c_{k, \sigma}^{\dagger} c_{k, \sigma}+\sum_{k, \alpha} \eta_{k} B_{k, \alpha}^{\dagger} B_{k, \alpha}
$$

such that

$$
E_{M F}=<0_{M F}|H| 0_{M F}>
$$

is minimum. $\mid 0_{M F}>$ is the direct product of a half filled Fermi sea of $c$-electrons and of a Fermi sea of berryons filled up to $x \equiv N_{B} / N$. Here, $x$ can be regarded as an adjustable variational parameter (although $H_{M F}$ is not truly variational, as it violates the constraints required by Eq. (16)). The precise value of $x$ is however immaterial, since the qualitative results we will find appear to be independent of $N_{B}$.

Direct substitution gives

$$
E_{M F}=-\frac{8 t N}{\pi^{2}} \sin \left(\frac{\pi}{2} x\right)
$$

which is minimum for $x=1$. The single particle excitation energies are

$$
\begin{aligned}
\epsilon_{k} & =-2 \theta \cos k \\
\eta_{k} & =-\frac{4 t}{\pi} \cos k,
\end{aligned}
$$

where $\theta \equiv \frac{2 t}{\pi} \sin (\pi x / 2)$ is the effective mean field hopping amplitude for $c$-electrons.

The next step is the determination of the first nontrivial correction to the mean field due to the actual interaction (40) between $c$-electrons and berryons. These corrections are achieved through an expansion in the interaction $\left(H-H_{M F}\right)$ around the free dynamics $H_{M F}$. For this purpose, we write the full many-body partition function at temperature $1 / \beta$ [15]:

$$
Z=Z_{M F}\left\langle\left\langle e^{-S}\right\rangle_{B}\right\rangle_{c} \approx Z_{M F}\left\langle e^{-<S>_{B}+\frac{1}{2}\left[<S^{2}>_{B}-<S>_{B}^{2}\right]+\ldots}\right\rangle_{c}
$$

with

$$
\begin{aligned}
Z_{M F} & =Z_{M F}^{c} Z_{M F}^{B} \\
S & =\int_{0}^{\beta} d \tau\left[H(c(\tau), \ldots B(\tau))-H_{M F}(c(\tau), \ldots B(\tau))\right]
\end{aligned}
$$




$$
\begin{aligned}
<O\left[c_{k \sigma}\right]>_{c} & =\int \frac{D\left[c_{k \sigma}^{\dagger} c_{k \sigma}\right]}{Z_{M F}^{c}} e^{-\int_{0}^{\beta} d \tau \sum_{k, \sigma} c_{k \sigma}^{\dagger}(\tau)\left(\partial_{\tau}+\epsilon_{k}\right) c_{k \sigma}(\tau)} O\left[c_{k \sigma}(\tau)\right] \\
<O\left[B_{k \alpha}\right]>_{B} & =\int \frac{D\left[B_{k \alpha}^{\dagger} B_{k \alpha}\right]}{Z_{M F}^{B}} e^{-\int_{0}^{\beta} d \tau \sum_{k, \alpha} B_{k \alpha}^{\dagger}(\tau)\left(\partial_{\tau}+\eta_{k}\right) B_{k \alpha}(\tau)} O\left[B_{k \alpha}(\tau)\right]
\end{aligned}
$$

where $O[$.$] is any operator, and$

$$
Z_{M F}^{c}=\int D\left[c_{k \sigma}^{\dagger} c_{k \sigma}\right] e^{-\int_{0}^{\beta} d \tau \sum_{k, \sigma} c_{k \sigma}^{\dagger}(\tau)\left(\partial_{\tau}+\epsilon_{k}\right) c_{k \sigma}(\tau)}
$$

and a similar expression for $Z_{M F}^{B}$.

Averaging over the non-interacting many body $B$-fields in the cumulant expansion in (46) leaves an effective hamiltonian operator for the $c$-electrons. That expansion contains a first term $<S>_{B}$ whose form is $\int_{0}^{\beta} d \tau \sum c_{k \sigma}^{\dagger}(\tau) c_{k \sigma}(\tau)$. It simply renormalizes the mean field parameters. The lowest order nontrivial action correction belongs to $-<S^{2}>_{B}$, having the form of an effective electron-electron interaction term

$$
S_{e f f}=\frac{1}{N} \int d \tau \int d \tau^{\prime} \sum_{\sigma, \sigma^{\prime}} \sum_{k_{1}, k_{2}, k_{3}, k_{4}} c_{k_{1} \sigma}^{\dagger}(\tau) c_{k_{2} \sigma}(\tau) c_{k_{3} \sigma^{\prime}}^{\dagger}\left(\tau^{\prime}\right) c_{k_{4} \sigma^{\prime}}\left(\tau^{\prime}\right) K_{k_{1}, k_{2}, k_{3}, k_{4}}\left(\tau-\tau^{\prime}\right)
$$

This term has a very simple significance. It corresponds to the exchange of a berryon particle-hole pair with singlet total pseudospin between the two electrons, as in the diagram of Fig. 6.

The imaginary time integration can be recast in a Matsubara frequency summation, in terms of a kernel

$$
\begin{aligned}
K_{k_{1}, k_{2}, k_{3}, k_{4}}\left(i \omega_{B}\right)= & \delta_{k_{1}+k_{3}, k_{2}+k_{4}} \frac{t^{2}}{2 N} \sum_{k} \cos \left(k_{1}+k\right) \cos \left(k_{3}+k+q\right) \\
& \frac{1}{\beta} \sum_{\omega_{n}}\left[-2 \tilde{g}_{k \uparrow}\left(\omega_{n}\right) \tilde{g}_{k \uparrow}\left(\omega_{n}+\omega_{B}\right)+\right. \\
& \left.\tilde{g}_{k \uparrow}\left(\omega_{n}\right) \tilde{g}_{k \downarrow}\left(-\omega_{n}-\omega_{B}\right) \tilde{g}_{k \downarrow}\left(\omega_{n}\right) \tilde{g}_{k \uparrow}\left(-\omega_{n}+\omega_{B}\right)\right],
\end{aligned}
$$

where $q:=k_{2}-k_{1}, \omega_{n}$ are fermionic Matsubara frequencies, and $\tilde{g}_{k \alpha}\left(\omega_{n}\right)$ is the free fermion propagator in Matsubara space as defined in [15]. The sum over the Matsubara frequencies can be performed to recast Eq. (50) in form

$$
\begin{aligned}
K_{k_{1}, k_{2}, k_{3}, k_{4}}\left(i \omega_{B}\right)= & \delta_{k_{1}+k_{3}, k_{2}+k_{4}} \frac{t^{2}}{2 N} \sum_{k} \cos \left(k_{1}+k\right) \cos \left(k_{3}+k+q\right) \\
& {\left[2 \Sigma\left(i \omega_{B} ; \xi_{k}, \xi_{k-q}\right)-\Sigma\left(i \omega_{B} ; \xi_{k},-\xi_{k-q}\right)-\Sigma\left(i \omega_{B} ;-\xi_{k}, \xi_{k-q}\right)\right] }
\end{aligned}
$$


where $\Sigma(z, a, b):=\left[f_{F}(a)-f_{F}(b)\right] /[z-(b-a)], f_{F}()$ are Fermi occupation factors and $\xi_{k}:=\eta_{k}-\mu=-\frac{4 t}{\pi}\left[\cos (k)-\cos \left(\frac{\pi x}{2}\right)\right]$ are the single-particle excitation energies for the berryons reduced by the corresponding chemical potential.

We would now like to extract physical conclusions from this calculation. Since we deal with an effective $1 \mathrm{D}$ electron system, we wish to use the calculated effective electron-electron scattering as a guide to understanding which one of the standard 1D Luttinger model fixed points will prevail.

In particular, for that model, an estimate of the forward and backward coupling constants $g_{1}$ and $g_{2}$ [13 will determine what kind of ground state to expect.

The pair scattering amplitude we have obtained is obviously time- (or frequency-) dependent, i.e., non-hamiltonian in nature. In this sense, straight identification with true hamiltonian parameters such as $g_{1}$ and $g_{2}$ [13] is not automatically correct. However, we see no physical reason preventing us from using our derived amplitudes as effective coupling constants so long as we stay sufficiently close to the Fermi surface.

We therefore identify

$$
g_{1} \propto K_{-k_{F}, k_{F}, k_{F},-k_{F}}\left(i \omega_{B}\right) \quad(q=\pi), g_{2} \propto K_{k_{F}, k_{F},-k_{F},-k_{F}}\left(i \omega_{B}\right) \quad(q=0) .
$$

Direct computation of $K$ for these special momenta gives:

$$
\begin{aligned}
& K_{-k_{F}, k_{F}, k_{F},-k_{F}}(z)=-\frac{t^{2}}{2 N} \int_{0}^{\pi} \frac{d k}{\pi} \sin ^{2}(k)\left[f_{F}\left(-\xi_{k}\right)-f_{F}\left(\xi_{k}\right)\right] \frac{2}{2 \xi_{k}-z} \\
& K_{k_{F}, k_{F},-k_{F},-k_{F}}(z)=-\frac{t^{2}}{2 N} \int_{0}^{\pi} \frac{d k}{\pi} \sin ^{2}(k)\left[f_{F}\left(-\xi_{k}\right)-f_{F}\left(\xi_{k}\right)\right] \frac{4 \xi_{k}}{4 \xi_{k}^{2}-z^{2}}
\end{aligned}
$$

Interestingly, these two couplings arise from different terms in the hamiltonian. The former is due to the $B^{\dagger} B, B B^{\dagger}$ terms in (40), whereas the latter to the $B^{\dagger} B^{\dagger}$ and $B B$ terms.

In the zero frequency limit $z \rightarrow 0$, the two quantities are negative and coincide. In terms of the Luttinger model phase diagram [13], this corresponds to a spin singlet superconducting state, which is therefore found to prevail. This is in very good agreement with the effective 
negative $U$ of the previous section, with the additional remark that the alternative possibility of charge-density waves is now explicitly ruled out.

Actually, the zero frequency limit is singular at zero temperature, when the Fermi functions become step functions and the $k$ integration diverges logarithmically for vanishing

frequency around $k_{F}^{B}$, the Fermi momentum for the berryons. In other words, in this approximation $g_{1}=g_{2}$ diverge as $\ln |z|$, at small $z$. This is a singular feature, due to our assuming the exchange of a bare, unrenormalized particle-hole pair as in Fig. 6. Higher order diagrams will modify that. More importantly, in presence of a finite pairing amplitude, for example, this divergence will disappear, due to a pairing gap in the berryon spectrum. There is in fact an exact symmetry between fermions and pseudofermions, and the two Cooper channels are also identical.

\section{DISCUSSION}

A very important property of our Berry phase-constrained tunnelling Hamiltonian is the fact that the constraint operates at the energy scale of the tunnelling matrix element t. Thus the pairing tendency induced by the constraints is not dictated directly by the strength of the intra-molecular electron-vibron coupling, but rather by the indirect effect of this coupling in the semi-classical limit on the relative phase space available for single electron tunnelling versus that for pair tunnelling. Thus the enforcing of the Berry phase constraint effectively separates the energy scale of the tunnelling, $t$, from that of the internal degrees of freedom of the constituent molecules.

In the case of $\mathrm{C}_{60}$ itself, the model is in too extreme a semi-classical limit to give a reasonable representation of the physics of $\mathrm{K}_{3} \mathrm{C}_{60}$ since, in that case, the dimensionless electron-vibron coupling strength $g$ is of order $\sim 0.4$, whereas the strong coupling limit where the Berry phase representation becomes useful is for $g \gtrsim 1$.

Nevertheless, the model does illustrate a new physical principle for superconductivity in strongly constrained systems. It is tempting to make an analogy with the physics of the 
$t-J$ model of interest for describing the physics of doped Mott insulators. In that case the hopping Hamiltonian my be re-written as

$$
H_{t}=-t \sum_{<i j>}\left(S_{i}^{+} c_{i \downarrow}^{\dagger} c_{j \downarrow} S_{j}^{-}+S_{i}^{-} c_{i \uparrow}^{\dagger} c_{j \uparrow} S_{j}^{+}+\text {h.c. }\right)
$$

where $S_{i}^{+}, S_{j}^{-}$are spin raising and lowering operators. A number of recent studies [16] suggest that pairing of holes close to the half-full insulating state occurs as a result of kinematical constraints in this model.

Although the present model does not have the ordered or quasi-ordered background of the antiferromagnetic state in the $t-J$ model, it does have the feature that pair tunnelling proceeds by each partner causing a vibron excitation when executing a tunnelling step, which then annihilate when the pair of carriers come together again on the same site. Similarly, in the $t-J$ model case, individual hole hopping is accompanied by a spin-flip, which can the be cancelled by the hopping of its partner.

It is of interest to consider whether our Berry phase considerations could also apply to the Chevrel-phase class of superconductors such as $\mathrm{LaMo}_{6} \mathrm{Se}_{8}$ or $\mathrm{PbMo}_{6} \mathrm{~S}_{y} \mathrm{Se}_{8-y}$ [17]. In these materials the $\mathrm{Mo}_{6} \mathrm{Se}_{8}$ cluster has a set of degenerate LUMO orbitals analogous to those in $\mathrm{C}_{60}$. Measurements of the doping dependence of $T_{c}$ indicate a sharp maximum as a function of doping in the unfilled LUMO shell. Thus there is the possibility of a general class of constraint-driven superconductors with distinctly different dependence of $T_{c}$ on material parameters than those of the conventional BCS-type electron-phonon superconductors [18].

Although, as stated above, we do not expect our model to be a realistic representation of the physics of $\mathrm{K}_{3} \mathrm{C}_{60}$, it would nevertheless be interesting to test experimentally whether the kind of electron-vibron coupling we have proposed could be observed in this compound. One way to do this would be through the two-vibron Raman spectrum. Our coupling mechanism would naturally lead to a direct electron-hole pair channel coupling to a pair of vibron modes. This channel would open up a gap of $2 \Delta$, in the vibron spectrum where $\Delta$ is the superconducting gap, on lowering the temperature of the material below $T_{c}$.

More generally, we observe that the pseudorotor Berry phase mechanism sketched here, 
ties together electron hopping with the hopping of quanta of orbital molecular angular momentum, which is unquenched in the free-molecule limit we start from. In the paired state orbital quanta are also paired, whereby the orbital excitation branch will also develop a gap at $q=2 k_{F}$. The gap will follow identically the superconducting gap at $T=T_{c}$. The staggered orbital susceptibility should therefore be maximum at $T_{c}$. In turn, the uniform $q=0$ orbital susceptibility may also develop a maximum, although weaker, via momentum nonconserving or local field effects. It is possible that orbital effects of this kind, even if weaker than suggested by this extreme picture, could be detectable, e.g., by NMR. In particular, the relaxation time $1 / T_{1}$ could be enhanced at low temperature, and peak up around $T_{c}$ due to large susceptibility fluctuations [19].

It would be of considerable interest to see if these new effect could be observed in $\mathrm{K}_{3} \mathrm{C}_{60}$, and $\mathrm{Rb}_{3} \mathrm{C}_{60}$. Encouragingly, in this latter compound, very recent NMR data [20] seem to indicate a behavior of the relaxation time which is anomalous precisely in the way suggested above. The anomaly at $T_{c}$, in particular, is seen in the $\mathrm{Rb}$ ion, but not on the carbon, as we would expect for a $\mathrm{C}_{60}$ orbital effect.

Finally, it is of interest to speculate that $T_{c}$ would be enhanced by doping our model system away from half-filling. Because of the nature of our pairing mechanism, the carriers would have more phase space for pairing if each partner in a pair could find many empty neighboring sites to hop on to before re-pairing. Thus the doping dependence of $T_{c}$ might be expected to have a maximum away from half-filling in systems for which this mechanism is driving the superconductivity. In this sense the case of half filling is probably the least favorable. There are indications from an exact solution for the 2-electron state that in model $\mathrm{EP}, U_{\text {eff }}$ is one order of magnitude more attractive near zero filling [21.

In the fullerides, exact half filling appears to be required by chemical stability 22. In the Chevrel systems, however, where continuous doping is feasible, one indeed finds a maximum of $T_{c}$ for a hole density close to one per molecular unit [17]. This corresponds to only $1 / 6$ filling of the narrow $\Gamma_{25}$ molecular band in that case [23]. A second observation is that the correlation length should tend to be short, of the order of the intermolecular distance $a$, 
since this is the scale where the energy gain takes place. In $\mathrm{K}_{3} \mathrm{C}_{60}$, this expectation is well borne out, with a correlation length of order $\equiv 2 a$.

Experimentally, it would be of interest to consider building new molecular solids where high-symmetry Jahn-Teller molecules can exchange electrons. Larger molecules may be better ones because of a weaker intramolecular Coulomb repulsion $U$. Relatively weak JT coupling may provide an additional favourable circumstance, since in that case the effective $\Omega$ is larger (although our treatment does not strictly apply there) and DJT quantum effects are more important. Both these conditions are met in the fullerides, but it might be possible to find other systems where they apply.

\section{CONCLUSIONS}

We have proposed a model for constrained tunnelling of charge carriers in a lattice of Berry phase molecules, inspired by the physics of the fullerides.

The general model (Eq. 22) is based on an electron-quantum rotor Hamiltonian which includes a (in principle infinite) manifold of vibron states on each site. Although we have been able to investigate the effects of a large number of vibron states on the pairing tendency, it is clear that the effect is strongest when only the lowest are important. For the extreme case where only the lowest vibron state is important $(S=1$ pseudospin model), both our numerical studies on small clusters (Section [II]) and our BCS-type mean field treatment (Section IV) indicate a strong pairing tendency for a half-filled band. The fact that the

model exhibits intrinsic pairing even in the presence of Hubbard repulsion $U$ of order the tunnelling matrix element $t$ is understood readily from the form of the model Hamiltonian (Eq. 1, 22). On integrating out the vibron degrees of freedom, we obtain an effective BCS attraction of order $t^{2} / W$, where $W$ is a vibron bandwidth of order $t$. Thus the Berry phase constraint leads to an electron-electron attraction whose energy scale is not directly related to the strength of the intra-molecular electron-vibron coupling in the semi-classical limit.

In physical terms, our model is based on the entanglement of orbital angular momentum 
of the individual molecules with electron hopping between molecules. Pairing of electrons is generated by an accompanying ("singlet") pairing of orbital momenta on neighbouring molecules, suggesting short correlation lengths in the order of the intermolecular spacing. It has been argued that this mechanism might be relevant also in such other molecular superconductors, such as the Chevrel compounds.

\section{ACKNOWLEDGMENTS}

It is a special pleasure to acknowledge many illuminating discussion with and much help

from G. Santoro and A. Parola, and also from M. Airoldi and M. Fabrizio. The sponsorship of NATO, through CRG 920828 and of EEC through Contract ERBCHRXCT420062, are gratefully acknowledged. 


\section{REFERENCES}

[1] M.V. Berry, Proc. R. Soc. Lond. A 392, 45 (1984).

[2] C. A. Mead, Rev. Mod. Phys. 64, 51 (1992).

[3] A. Auerbach, Phys. Rev. Lett. 72, 2931 (1994); A. Auerbach, N. Manini, and E. Tosatti, Phys. Rev. B 49, 12998 (1994).

[4] E. Tosatti, Invited paper, Euroconference on Superconductivity, Pisa, 21-25 Jan. 1993 (unpublished).

[5] G. Delacrétaz, E. R. Grant, R. L. Whetten, L. Woste, and J. W. Zwanziger, Phys. Rev. Lett. 56, 2598 (1986).

[6] R. Englman, The Jahn Teller Effect in Molecules and Crystals (Wiley, London, 1972).

[7] H. C. Longuet-Higgins, Adv. Spect. 2429 (1961), and references therein; G. Herzberg and H. C. Longuet-Higgins, Discuss. Faraday Soc. 35, 77 (1963); H. C. Longuet-Higgins, Proc. Roy. Soc. Lond. A 344, 147 (1975).

[8] H. Uehara, J. Chem. Phys. 45, 4536 (1966).

[9] For a more general lattice symmetry, there will be also terms $c_{x}^{\dagger} c_{y}$ but we stick for convenience to the simpler high symmetry case for the time being.

[10] J.C. Slater and G.F. Koster, Phys. Rev. 94, 1498 (1954); F. Bassani and G. Pastori Parravicini, Electronic States and Optical Transitions in Solids (Pergamon, Oxford, 1975), Chap. 3.

[11] Although $L^{+}$and $L^{-}$commute in the full rotor space, it is easy to verify that $\left[S^{+}, S^{-}\right]=$ $S^{z}$, as appropriate in a spin algebra.

[12] For a better understanding of the large $N$ thermodynamic limit, we are presently considering finite-size scaling studies of this model. 
[13] J. Sólyom, Adv. Phys. 28, 201 (1979).

[14] E.H. Lieb, Proceedings of the conference Advances in Dynamical Systems and Quantum Physics, Capri, 1993 (World Scientific, Singapore, 1993)

[15] J. W. Negele, and H. Orland, Quantum Many-Particle Systems (Addison-Wesley, Redwood City CA, 1988), Ch. 2.

[16] J.H. Xu, C.S. Ting, and T.K. Lee, Phys. Rev. B 43, 8733 ( 1991); O.P. Sushkov, Phys. Lett. A 162, 199 (1992); M. Boninsegni, and E. Manousakis, Phys. Lett. A 178, 175 (1993); P. Wrobel, and R. Eder, Phys. Rev. B 49, 1233 (1994).

[17] R. Chevrel in Superconductor Materials Science, edited by S. Foner and B. Schwartz (Plenum, New York, 1981), p. 685.

[18] Y.J. Uemura, L.P. Le, G.M. Luke, B.J Sternlieb, W.D. Wu, J.H. Brewer, T.M. Riseman, C.L. Seaman, M.B. Maple, M. Ishikawa, D.G. Hinks, and J. Jorgensen, Phys. Rev. Lett. 66, 2665 (1991).

[19] A. Rigamonti, private communication.

[20] G. Zimmer, M. Helmle, M. Mehring, J. Reichenbach, F. Rachdi, and J.E. Fischer, preprint (1994).

[21] G. Santoro and A. Parola, private communication.

[22] The insulating compounds $\mathrm{K}_{4} \mathrm{C}_{60}$, and $\mathrm{Rb}_{4} \mathrm{C}_{60}$, on the other hand, cannot really be thought as the result of doping $\mathrm{K}_{3} \mathrm{C}_{60}$, and $\mathrm{Rb}_{3} \mathrm{C}_{60}$ away from half filling. We have in fact suggested that their insulating behavior should be of Jahn-Teller nature [24].

[23] L.F. Mattheiss and C.Y. Fong, Phys. Rev. B 15, 1760 (1977).

[24] N. Manini, E. Tosatti, and A. Auerbach, Phys. Rev. B 49, 13008 (1994). 


\section{FIGURES}

FIG. 1. Structure factors $S_{S C}(q)$ (32) and $S_{S D W}(q)$ (33) for the Hubbard model (6 sites ring), as a function of the dimensionless parameter $U / t$. In the upper panel periodic boundary conditions (PBC) are applied to indexes, so that for $U=0$ the ground state is nondegenerate ("closed shell"), while in the lower panel antiperiodic boundary conditions (ABC) make the noninteracting ground state degenerate ("open shell"). In panel a) $q=0$, while in panel b) $q=\pi / N$, as appropriate to the boundary conditions applied. Solid dots mark crossings, where the switching from superconductivity to spin-density waves takes place.

FIG. 2. Structure factors $S_{S C}(32)$ and $S_{S D W}(33)$ for the EP model, plotted as a function of $U / t$. In panel a) we report the result for the closed shell case, while panel b) has the result for the open shell case, as discussed in the text. Solid dots mark crossings defining $U^{*}$, indicating that superconductive pairing prevails even at positive $U$.

FIG. 3. Structure factors $S_{S C}(32)$ and $S_{S D W}$ (33) for the ER model $(N=4,6)$, plotted as a function of $U / \Omega$, with $t=1$. In panel a) we report the result for the closed shell case, while panel b) has the result for the open shell case. $E_{c u t}=6$ for $N=4$, and $E_{c u t}=3$ for $N=6$. Solid dots mark crossings defining $U^{*}$, again indicating pairing even at positive $U$.

FIG. 4. The effective Hubbard term $U_{\text {eff }}(35)$ for the ER model $(N=4,6)$, plotted as a function of $t / \Omega$. The $N=4 \mathrm{ABC}$ and $N=6 \mathrm{PBC}$ are closed shells, while $N=4 \mathrm{PBC}$ and $N=6 \mathrm{ABC}$ are open shell cases. $E_{\text {cut }}=8$ for $N=4$, and $E_{\text {cut }}=4$ for $N=6$. For $t / \Omega<<1$, the slope of $U_{\text {eff }}$ coincides with $U_{\text {eff }} / t$ of the EP model (Fig. 2). For $t / \Omega>>1$, the dynamical Jahn-Teller effects are suppressed, whence $U_{\text {eff }} \rightarrow 0$.

FIG. 5. The effective Hubbard term $U_{\text {eff }}(35)$ for the EP model $(N=4,6$, in closed shells) with the addition of the term (36), describing $t_{x} \neq t_{y}$, plotted as a function of $t / t^{\prime}$, at fixed $t+t^{\prime}=t_{x}=1$. The effective interaction survives everywhere except at the isolated point $t / t^{\prime}=1$, corresponding to the unphysical case $t_{y}=0$ (or $\left.t_{x}=0\right)$. 
FIG. 6. The second order effective interaction between electrons is due to the exchange of a pair of berryons. 
Manini, Tosatti, and Doniach, FIG 1
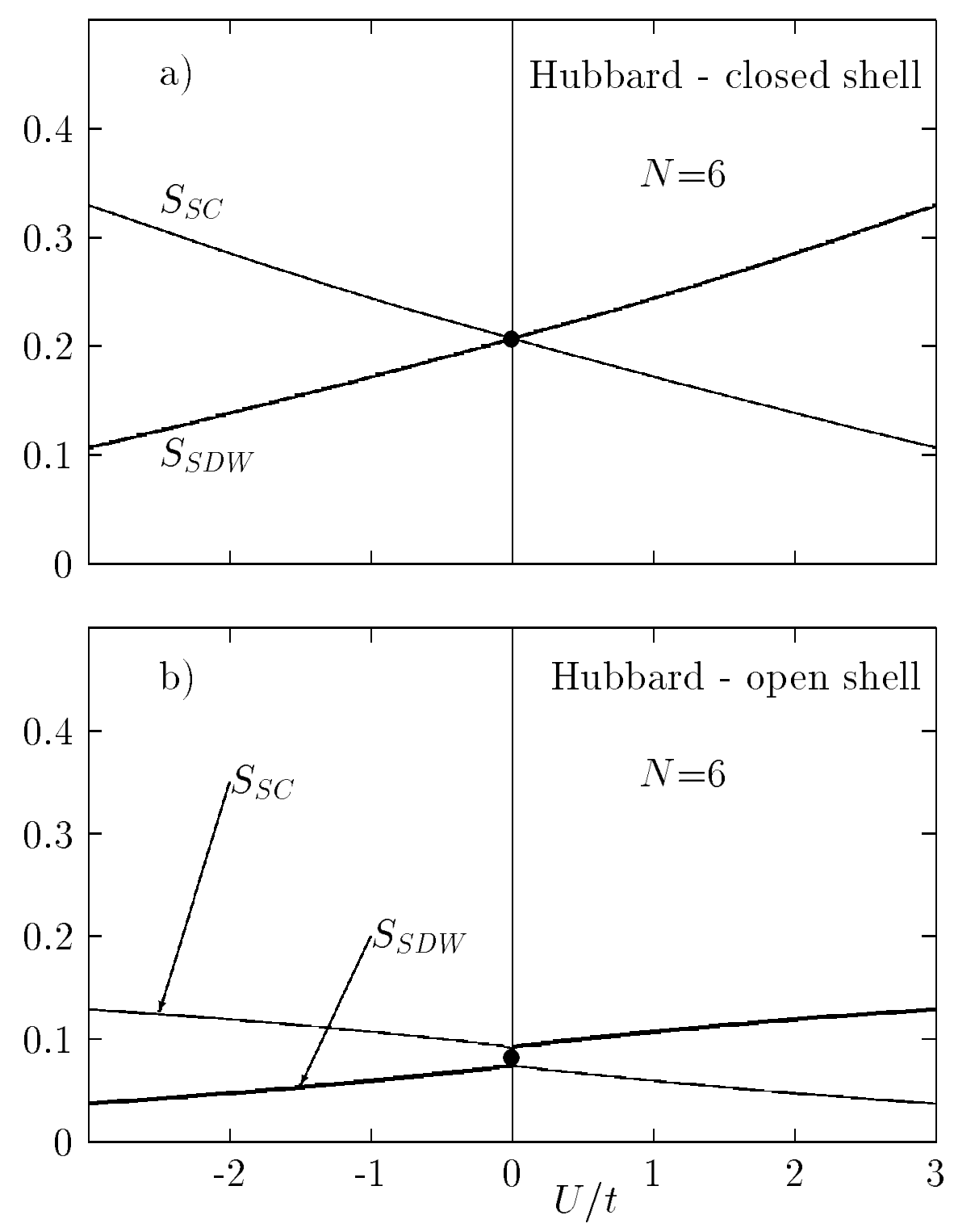
Manini, Tosatti, and Doniach, FIG 2
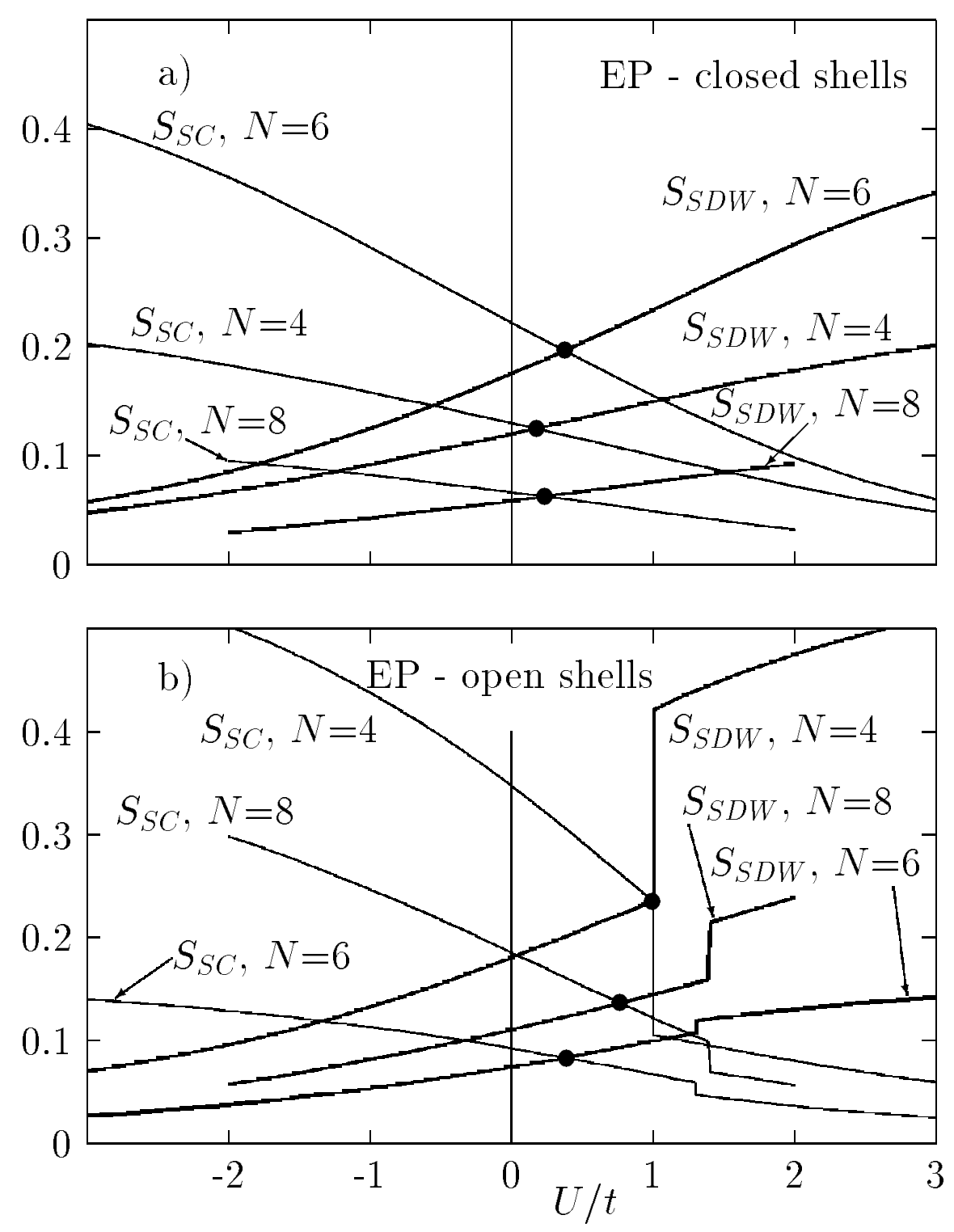
Manini, Tosatti, and Doniach, FIG 3
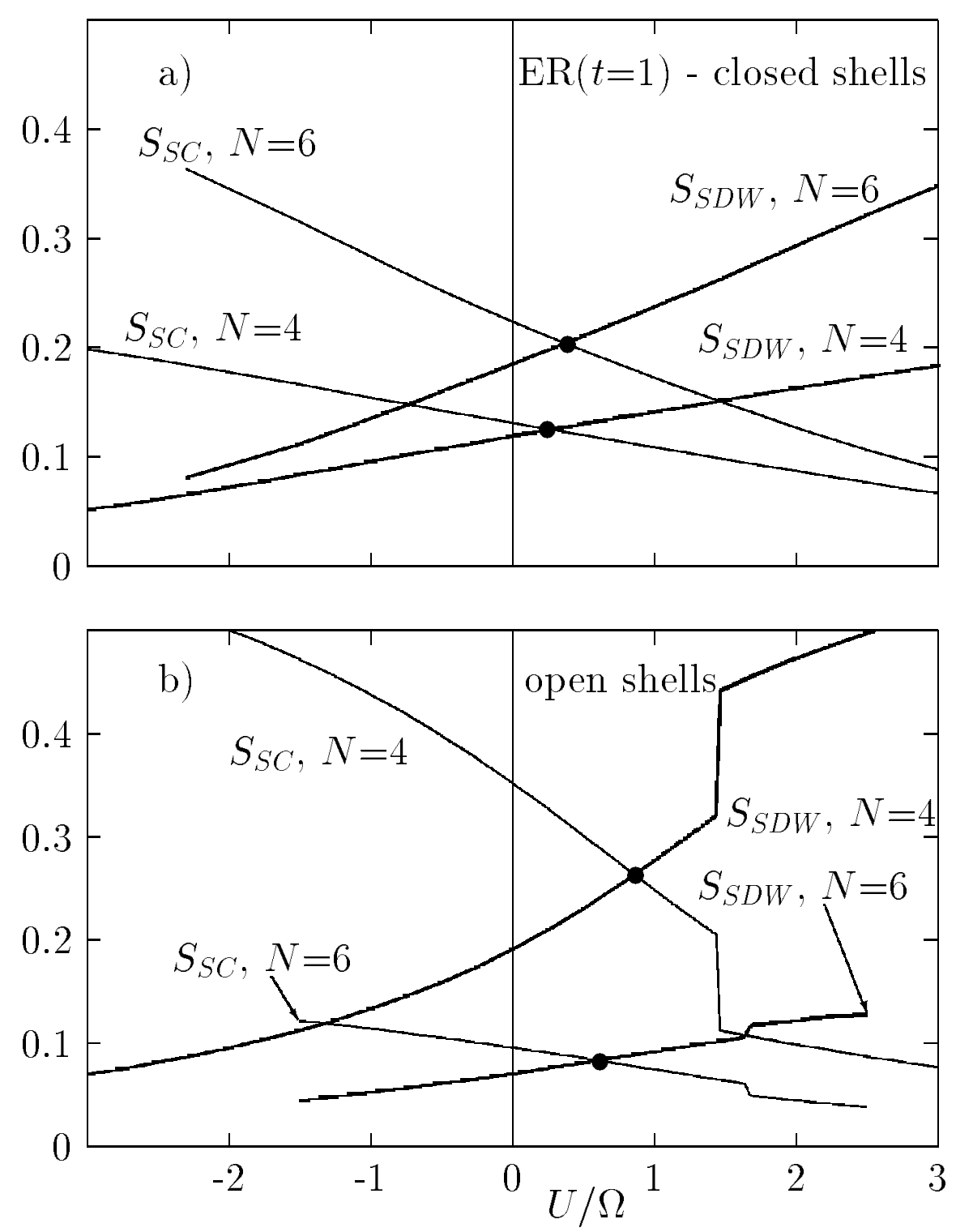
Manini, Tosatti, and Doniach, FIG 4

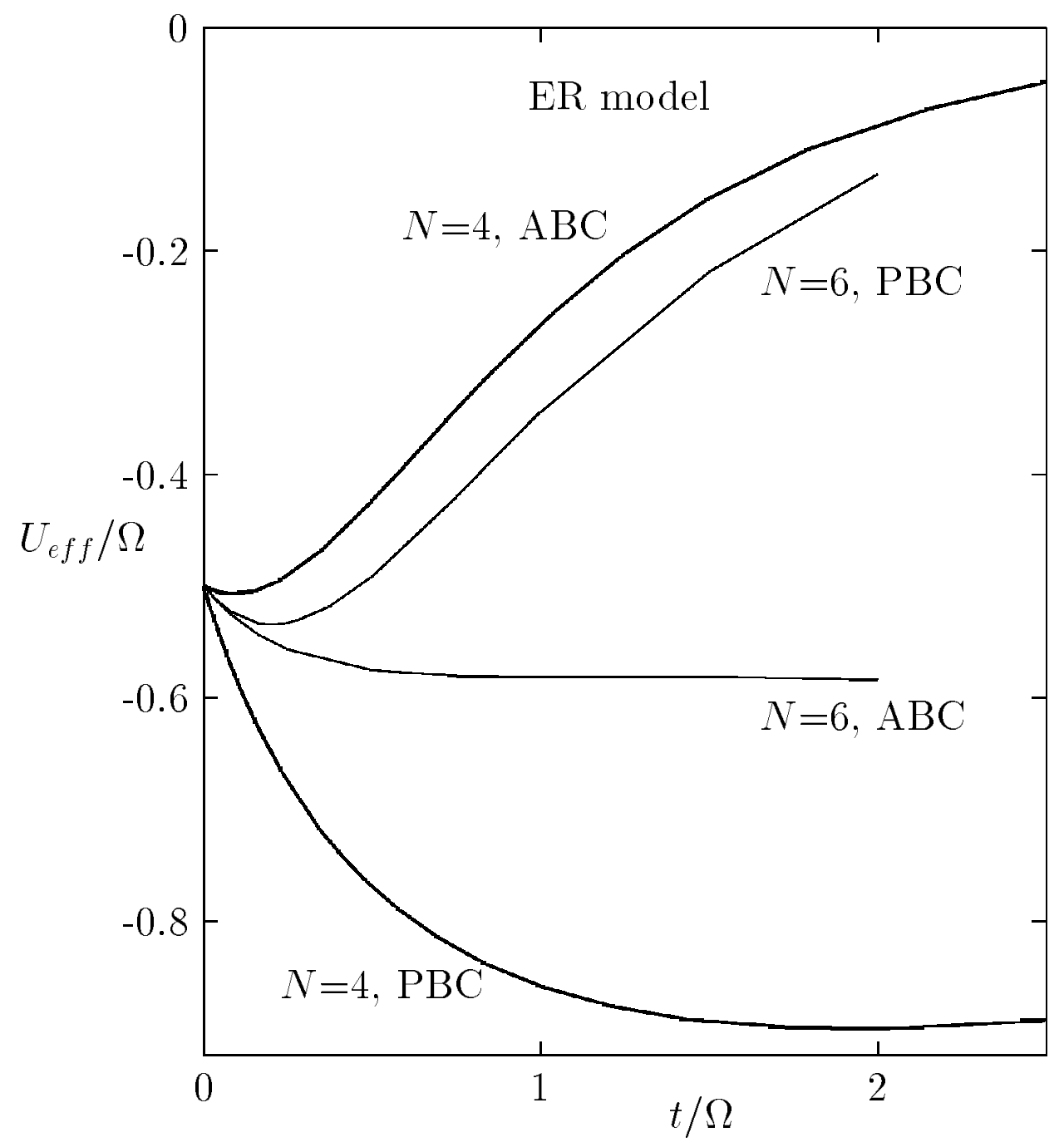


Manini, Tosatti, and Doniach, FIG 5

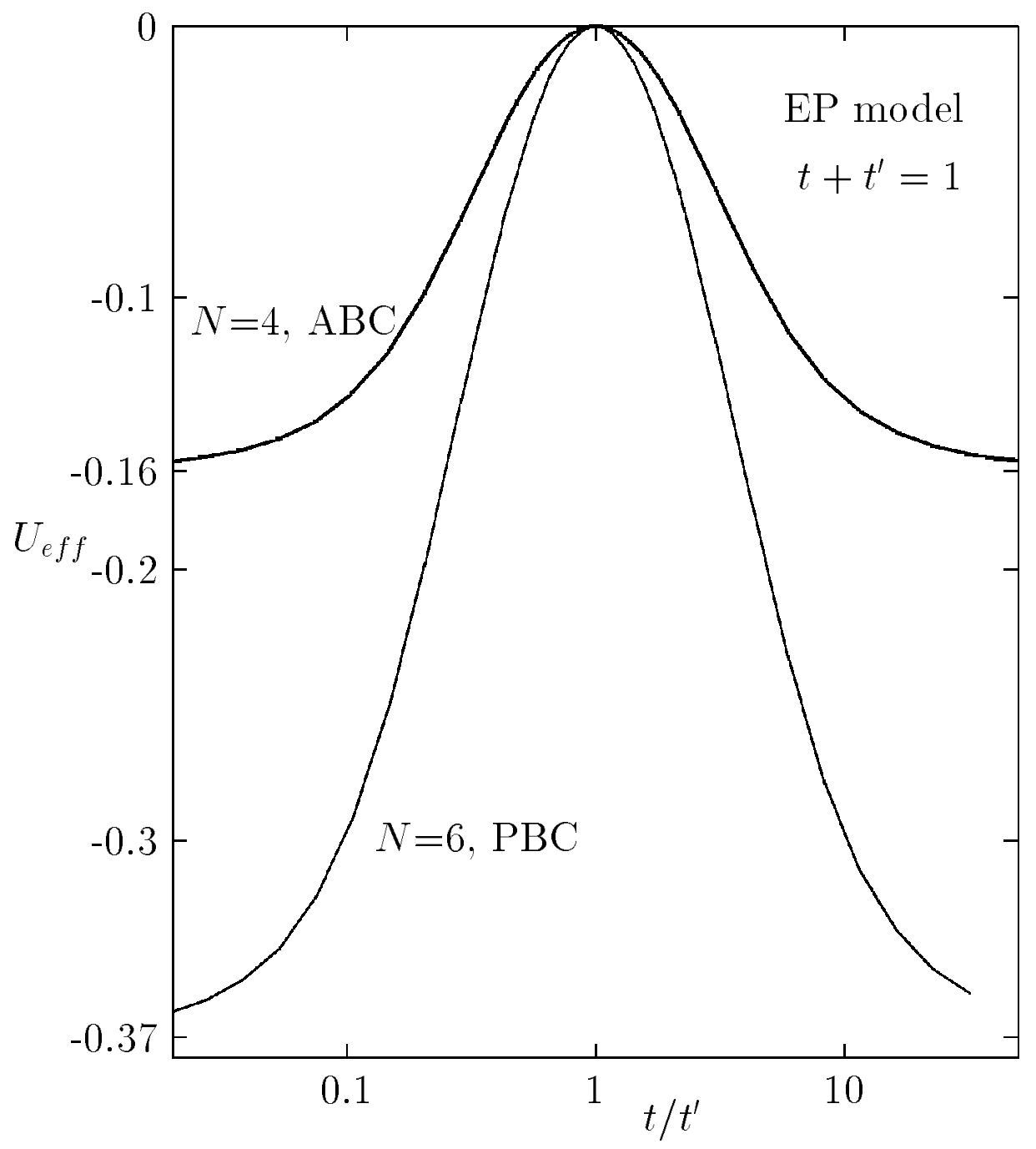


Manini, Tosatti, and Doniach, FIG 6

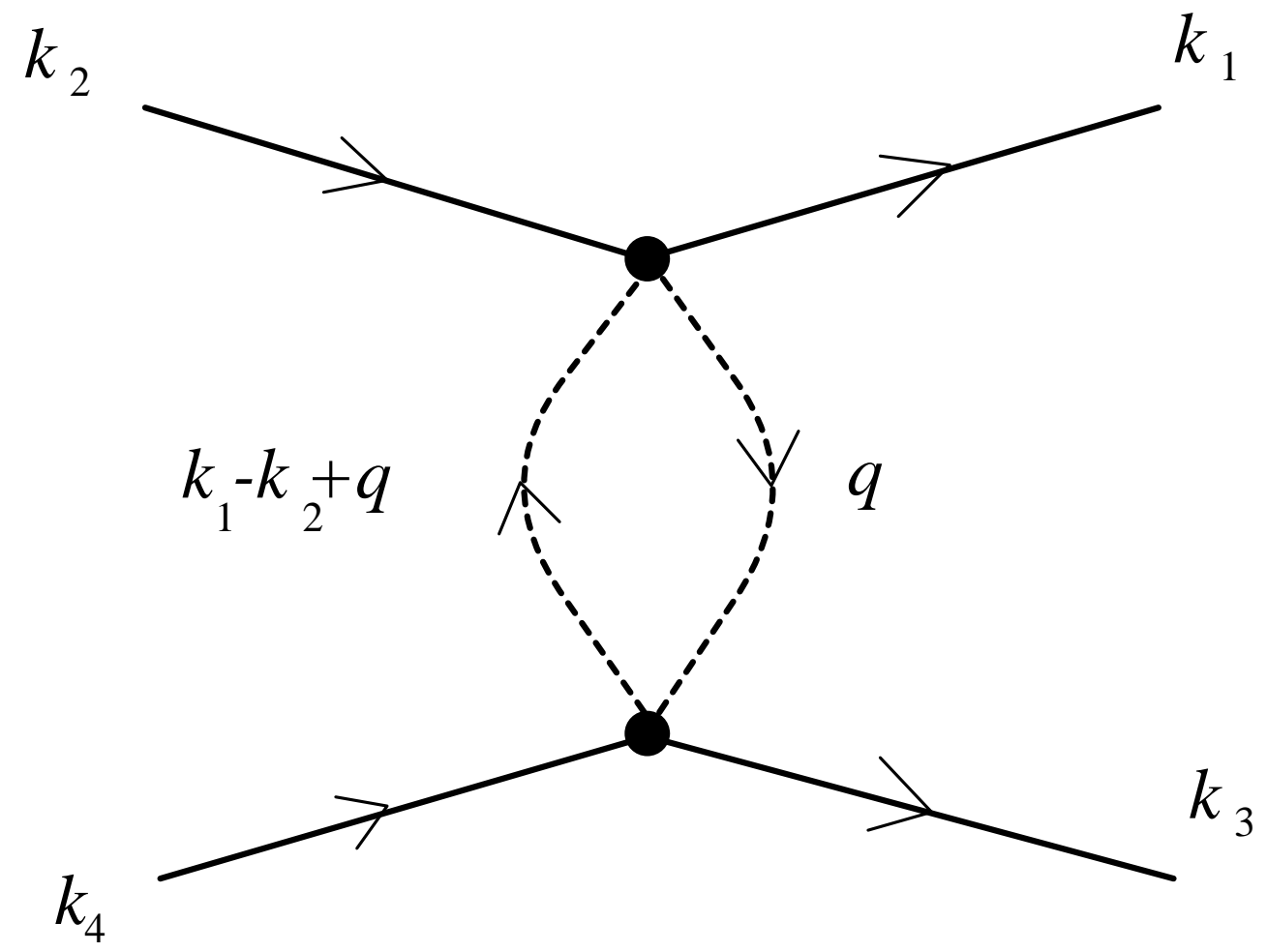

